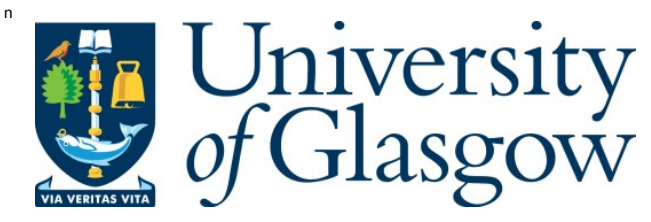

Ceriotti, M ., Harkness, P., and M CR obb, M . (2014) Synchronized orbits and oscillations for free altitude control. Journal of Guidance, Control and Dynamics, 37 (6). pp. 2062-2066. ISSN 0731-5090

Copyright $\odot 2014$ AIA A

A copy can be downloaded for personal non-commercial research or study, without prior permission or charge

Content must not be changed in any way or reproduced in any format or medium without the formal permission of the copyright holder(s)

http://eprints.gla.ac.uk/93109/

Deposited on: 23 A pril 2014

Enlighten - Research publications by members of the U niversity of Glasgow http://eprints.gla.ac.uk 


\title{
Synchronized orbits and oscillations for free altitude control
}

\author{
Matteo Ceriotti, ${ }^{*}$ Patrick Harkness ${ }^{\dagger}$ and Malcolm McRobb \\ University of Glasgow, Glasgow, G12 8QQ, United Kingdom
}

\section{Nomenclature}

$\begin{array}{ll}A & \text { Sail area, } \mathrm{m}^{2} \\ b & \text { Sail baseline, } \mathrm{m} \\ \mathrm{CM} & \text { Centre of mass } \\ \mathrm{CP} & \text { Centre of pressure } \\ d & \text { Heliodynamic static margin, } \mathrm{m} \\ \mathbf{f} & \text { Force vector, } \mathrm{N} \\ I & \text { Rotational inertia, } \mathrm{kg} \mathrm{m}^{2} \\ l & \text { Boom length, } \mathrm{m} \\ l_{\text {bus }} & \text { Spacecraft bus reference length, } \mathrm{cm} \\ m & \text { Mass of the system, } \mathrm{kg} \\ m_{\text {bus }} & \text { Spacecraft bus mass, } \mathrm{kg} \\ P_{\text {sun }} & \text { Solar radiation pressure, } 4.56 \times 10^{-6} \mathrm{~N} / \mathrm{m}^{2} \\ \mathbf{r}_{\mathrm{s}} & \text { Sun vector, km } \\ \mathbf{r}_{\mathbf{s}}{ }^{(\mathbf{E C I})} & \text { Sun vector in ECI frame, km } \\ t & \text { Time, hours or days } \\ T & \text { Period of an oscillation, } \mathrm{s} \\ U & \text { Rotational potential energy of the system, J } \\ \delta_{s} & \text { Declination of the sun on the spacecraft orbital plane, deg } \\ \varepsilon & \text { Obliquity of the ecliptic, } 23.5 \text { deg } \\ \eta & \text { Sail efficiency } \\ \theta & \text { Rotation about } x \text {-axis, deg } \\ \lambda & \text { Longitude, deg } \\ \tau & \text { Torque, Nm } \\ \omega & \text { Angular velocity, rad } / \mathrm{s}\end{array}$

\section{Introduction}

The concept of using a deployed structure to impart aerostability to spacecraft operating in the extremely rarefied atmosphere of the lowest Earth orbits is well established [1-3], and recently the cognate opportunities presented by the heliostability of similar geometries in higher orbits has been discussed [4]. Aerostability has also been long associated with the ability to maintain an aerodynamic force vector such as lift in the absence of active control, and heliostability can similarly be used to generate a steady force vector. This can achieved through the very familiar solar sail.

Studies in the literature have considered planet-centered solar sails, including optimal control laws [5, 6], orbit raising [7] and escape strategies [8]. In all these studies, however, an active attitude control system is required to steer the sail according to the control law.

\footnotetext{
${ }^{*}$ Lecturer, School of Engineering, James Watt Building South, Glasgow G12 8QQ, United Kingdom. AIAA Member.

$\dagger$ Lecturer, School of Engineering, James Watt Building South, Glasgow G12 8QQ, United Kingdom. AIAA Member.

$\$$ Research Assistant, School of Engineering, James Watt Building South, Glasgow G12 8QQ, United Kingdom.
} 
Conversely, a heliostable sail will naturally oscillate about the sun vector if disturbed, and the direction of the force vector will also oscillate. If these oscillations are synchronized with an orbital path the direction of the force vector will also oscillate once per orbit and thus can be set to point approximately but consistently in a particular direction, such as along the flight vector or towards zenith. This means that, once an appropriate behavior is initiated, a force can be generated that can adjust the altitude of a spacecraft with very little energy expenditure and no propellant whatsoever.

However, as orbital parameters change and the sun moves along the ecliptic, the synchronization of the heliostable oscillation and the progress along each orbit must be managed. This can be achieved by altering the period of the oscillation using changes in sail geometry (area or center of pressure), changes to the rotational inertia of the spacecraft [9], or changes to the reflectivity of the solar sail [10]. The latter option is particularly attractive because changing the pattern of reflectivity, perhaps using liquid-crystal panels [11], can roll the spacecraft to manage the plane of the heliostable oscillation and, secondly, can superimpose a time-variation on the restoring torque to either increase or diminish the amplitude of the excursions. This paper focuses in particular on the former method, showing how synchronous attitude/orbit oscillations can be exploited to increase the semi-major axis of a circular, equatorial orbit.

\section{Spacecraft}

The spacecraft is modeled by a bus from which a flat, triangular sail is deployed between two extensible booms of length $l$. A body reference frame is defined as in Fig. 1 . The center of pressure (CP) of the sail is at $2 / 3$ of the height of the sail itself, while the center of mass $(\mathrm{CM})$ of the spacecraft is close to the center of the spacecraft bus, at the origin of the body frame.

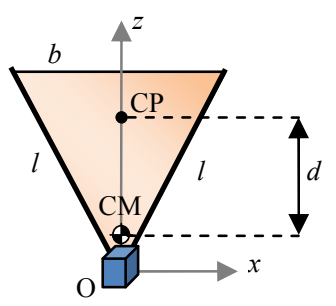

Fig. 1 Spacecraft configuration and body coordinate frame.

The force acting on the sail in the $y$-direction, which can be imagined applied at the CP, due to SRP is [12] (pp. 39-40):

$$
\mathbf{f}= \pm 2 \eta P_{\text {sun }} A\left(\hat{\mathbf{y}} \cdot \hat{\mathbf{r}}_{s}\right)^{2} \hat{\mathbf{y}}
$$

The sign is chosen depending whether the sun illuminates one side or the other of the sail. $\mathbf{r}_{s}$ is the sun vector, representing the direction of the sun in body axes, and $P_{\text {sun }}$ is the SRP of the sun, approximately $4.56 \times 10^{-6} \mathrm{~N} / \mathrm{m}^{2}$ at Earth distance. 
Finally, $\eta=0.85$ is the efficiency of the solar sail material in terms of reflectivity, ranging from 0.5 (full absorption) to 1 (full specular reflection). Note that diffraction and other effects are not explicitly considered here, but included in the efficiency $\eta$. Calling $d$ the heliodynamic static margin, i.e. distance between the $\mathrm{CP}$ and the $\mathrm{CM}$, the only component of the torque with respect to the $\mathrm{CM}$ is

$$
\tau_{x}=-d f
$$

\section{Attitude motion}

The attitude motion of the spacecraft is modeled through the standard Euler equations for a rigid body [13] (pp. 153156), forced by the torque in Eq. (2). If the spacecraft is released such that the sail is initially deflected by an angle $\theta_{0}$ around the $x$ axis (see Fig. 2) it will start an undamped oscillatory motion under the influence of the SRP with the neutral position aligned with the sun itself. The motion, however, is not purely harmonic, because the forcing term is not simply $\propto \theta$, but rather $\propto \sin ^{2}(\theta)$, according to Eq. (1). Fig. 2 shows the oscillation around the $x$-axis in the plane of the oscillation (a), and also in the plane perpendicular to it as defined by the $x$-axis and the sun vector (b). Note that the sun, in general, does not lie in the plane of oscillation, but it is tilted by $\delta_{s}$ out of that plane.

a)

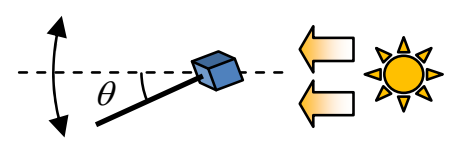

b)

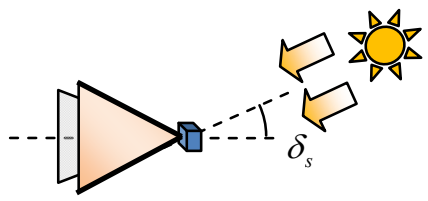

Fig. 2 Attitude oscillation around $x$-axis. (a) Oscillation plane view; (b) Side view.

\section{A. Period}

The period of one oscillation of the sailcraft can be found in the following way. First of all, we notice that the attitude motion is a conservative system. We can therefore write the expression for the rotational total energy:

$$
\frac{1}{2} I \dot{\theta}^{2}-\frac{1}{2} I \dot{\theta}_{0}^{2}=U(\theta)-U\left(\theta_{0}\right)
$$

In this equation, it is assumed that the sun's direction is inertially fixed. This is a valid approximation if the apparent rotation of the sun due to the Earth's orbital motion is slow with respect to the period of the oscillation, as will be the case here and as shall be further discussed in Section V. We also assume the spacecraft is at rotational rest when released, hence $\dot{\theta}_{0}=0$. The potential $U(\theta)$, considering $\theta=0$ as reference (sail aligned with the sun direction), can be written as: 


$$
U(\theta)=\int_{0}^{\theta} \tau_{x}\left(\theta^{\prime}\right) d \theta^{\prime}=2 \eta P_{s} A d \cos ^{2}\left(\delta_{s}\right) \int_{0}^{\theta} \sin ^{2}\left(\theta^{\prime}\right) \operatorname{sgn}\left(\theta^{\prime}\right) d \theta^{\prime}
$$

The term $\cos ^{2}\left(\delta_{s}\right)$ takes into account the declination of the sun out of the plane of oscillation. It results:

$$
U(\theta)= \pm 2 \eta P_{s} A d \cos ^{2}\left(\delta_{s}\right)\left[\frac{\theta}{2}-\frac{1}{4} \sin 2 \theta\right]
$$

in which the sign is chosen depending on $\theta \geq 0$ or $\theta<0$ respectively. This can also be written as:

$$
U(\theta)=2 \eta P_{s} A d \cos ^{2}\left(\delta_{s}\right)\left|\frac{\theta}{2}-\frac{1}{4} \sin 2 \theta\right|
$$

When the oscillation reaches its maximum amplitude, $\theta_{0}$, the angular velocity is null, i.e. $\dot{\theta}_{0}=0$. The value of $\theta_{0}$ is also known, because it corresponds to the initial condition in which the satellite is released. Hence we can exploit the conservation of energy between the initial condition and an arbitrary point:

$$
\frac{1}{2} I \dot{\theta}^{2}=U\left(\theta_{0}\right)-U(\theta)=2 \eta P_{s} A d \cos ^{2}\left(\delta_{s}\right)\left[\left|\frac{\theta_{0}}{2}-\frac{1}{4} \sin 2 \theta_{0}\right|-\left|\frac{\theta}{2}-\frac{1}{4} \sin 2 \theta\right|\right]
$$

This equation can be solved for $\dot{\theta}$ to give:

$$
\dot{\theta}=\sqrt{\frac{2 \eta P_{s} A d \cos ^{2}\left(\delta_{s}\right)}{I}} \sqrt{\left|\theta_{0}-\frac{1}{2} \sin 2 \theta_{0}\right|-\left|\theta-\frac{1}{2} \sin 2 \theta\right|}
$$

Noting that $\dot{\theta}=\frac{d \theta}{d t}$, we can separate the variables:

$$
d t=\sqrt{\frac{I}{2 \eta P_{s} A d \cos ^{2}\left(\delta_{s}\right)}} \frac{d \theta}{\sqrt{\left|\theta_{0}-\frac{1}{2} \sin 2 \theta_{0}\right|-\left|\theta-\frac{1}{2} \sin 2 \theta\right|}}
$$

The period can be computed as:

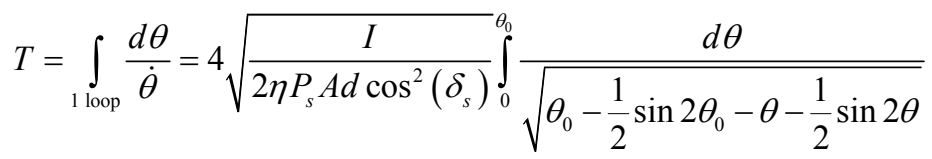

where the period of one full oscillation is found multiplying by four a quarter-period and the absolute values were removed because, without loss of generality, we can choose $\theta_{0}>0$. The integral is solved numerically to find $T$.

Note the dependence of $T$ on $\sqrt{I / \eta d A}$ : as noted before, the period can be changed varying the inertia $I$, the static margin $d$, the area $A$ or the reflectivity through $\eta$. In particular, increasing the length of the sail booms will produce a greater $I$, but it will also increase $d$ (if the sail mass is considerably lighter than the bus mass) and $A$. These two effects act against each 
other with respect to period change. Using a pair of opposed extensible booms with tip masses would not alter $d$, but only $I$; and using one extensible boom only in $+z$ would simultaneously increase $I$ and decrease $d$.

\section{Orbital motion}

For the orbital motion of the spacecraft, we consider the action of the gravity of the Earth (considered a point mass with planetary constant $\mu=3.986 \cdot 10^{5} \mathrm{~km}^{3} \mathrm{~s}^{-2}$ ) and the acceleration $\mathbf{f} / m$ due to SRP. The integration of the differential equations of motion is performed using Gauss' variational equations (Ref. [14], pp. 488-489). Note that these equations require the knowledge of the attitude of the spacecraft, as the acceleration must be transformed from body frame to orbit frame. Therefore the orbital motion is coupled with the attitude motion and, for this reason, the two dynamics are coupled and integrated together.

The direction of the sun, with respect to an Earth-centered inertial frame, is given by:

$$
\hat{\mathbf{r}}_{s}^{(E C I)}=\left[\begin{array}{lll}
\cos \lambda & \sin \lambda \cos \varepsilon & \sin \lambda \sin \varepsilon
\end{array}\right]^{T}, \quad \lambda=\lambda_{0}+t \dot{\lambda}
$$

where $\lambda$ is the longitude of the sun, $\dot{\lambda}$ is the angular velocity of the Earth around the sun (assumed constant at one revolution per year), and $\varepsilon=23.5^{\circ}$ is the obliquity of the equator on the ecliptic plane. By choosing $\lambda_{0}=0$, then at time $t=0$ the sun is at the vernal equinox in the equatorial plane.

\section{Control law}

We aim now at coupling the orbital motion on a circular orbit with the oscillatory attitude motion explained in Section III to show that is it possible to obtain a net change in semi-major axis in each orbit. According to Gauss' equations [14], a change in semi-major axis is obtained providing an acceleration tangential to the orbit, and we assume that the spacecraft is released such that plane of oscillation in Fig. 2 coincides with the orbital plane (thus $\delta_{s}$ is the declination of the sun over the orbital plane). If the sail oscillation is synchronized with the orbital motion (i.e. so that they have same period), and the phase between the two is chosen such that the maximum angular displacement is reached when the spacecraft is along the sun-Earth line, then the sail provides a tangential acceleration component which has its maximum near the sun-Earth line and its null near the axis perpendicular to it (see Fig. 3). In addition, there is also a component of the acceleration that is normal to the orbit. In the short term, the effect is to change the eccentricity and the anomaly of pericentre of the orbit, but the net change over one year is almost negligible due to the fact that the sun-Earth line rotates one full revolution in that time. 
a)

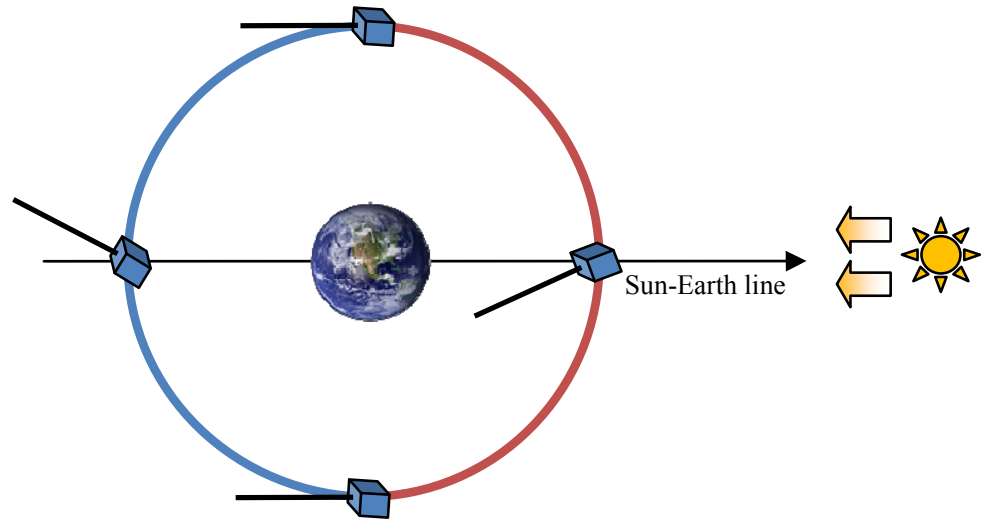

b)
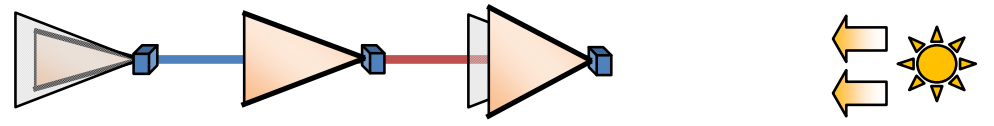

Fig. 3 Semi-major axis change. (a) Orbital plane view, the spacecraft orbits anticlockwise; (b) Side view.

Note that, as the sun-Earth line rotates, the oscillation will passively stay centered around the sun-Earth line direction because of the heliostability of the sail, and because the system is conservative, the oscillation is undamped.

However, a control is needed to maintain the phase of the attitude oscillation synchronous with the angular position on the orbit with respect to the sun-Earth line. In addition, the period of the oscillation has to be adjusted such that it matches the period of the orbit, which is increasing in time following the increase in semi-major axis. Finally, the declination of the sun over the orbital plane changes according to Eq. (4). This does not generate any acceleration out of the orbital plane, but it does however change the period of the oscillation, following the dependence on $\delta_{s}$ of Eq. (3).

We assume that we can change the moment of inertia around the oscillation axis (i.e. $x$ ), for example by extending a boom with a tip mass, and show that it is possible to fully control the oscillation of the spacecraft, and thus the orbital change, without any other actuator.

The control algorithm we apply is discrete and based on the consideration that, when the orbital angular position of the spacecraft is aligned with the sun-Earth line, the attitude oscillatory motion shall be at its maximum displacement and its angular velocity equal to zero. In particular, the angular velocity is crossing zero monotonically. Considering a control proportional to the state error, the moment of inertia can be changed according to the following law, at each orbital passage across the sun-Earth line (on the sun side):

$$
\Delta I_{x}=-k \omega_{x}
$$


where $k>0$ is the control gain. In fact, if $\omega_{x}>0$, the oscillation has not completed one period yet, and therefore the moment of inertia shall be reduced, such that the next period of the oscillation will be shorter, and vice-versa.

We assume that the change in moment of inertia is instantaneous. The initial moment of inertia required is computed, given the geometry of the spacecraft and its initial angle, inverting Eq. (3).

Note that in absence of perturbations the oscillatory motion of the spacecraft will stay on the orbital plane. However, it is realistic to predict that external perturbing torques will require some mild control to maintain the oscillation in this plane, as well as to avoid any unwanted rotation around the $z$-axis. This can be achieved, for example, with appropriate differential changes of the reflectivity of part of the sail, using the aforementioned electrically-controlled liquid crystals and, perhaps, suitable rotational dampers. The study of these perturbations and their control is out of the scope of this paper, and in the following we assume that the oscillation is unperturbed and purely around the spacecraft $x$-axis and in the orbital plane.

\section{A. Results}

We assume the spacecraft bus is a cube of $l_{\text {bus }}$ side and mass $m_{b u s}$, uniformly distributed. The sail is considered a flat triangle with uniform mass per unit area of $50 \mathrm{~g} / \mathrm{m}^{2}$, which also takes into account the booms. The satellite is released on a 1000-km-altitude circular equatorial orbit, such that the atmospheric effects can be neglected, on the sun-Earth line, with an initial angular displacement of $\theta_{0}=30^{\circ}$ with respect to the same. Small values for eccentricity and inclination are used to prevent singularities in the equations of motion. Spacecraft and sail masses and dimensions are given in Table 1 for two different spacecraft configurations.

Considering spacecraft 1 as the baseline design, Fig. 4 shows the evolution of semi-major axis and eccentricity over one year. The second spacecraft, due to the larger area-to-mass ratio, enables a higher semi-major axis gain (about $35 \mathrm{~km} / \mathrm{year}$ ) compared to the first configuration ( $20 \mathrm{~km} /$ year). The eccentricity remains substantially unchanged.

Fig. 5 (b) is a magnification of Fig. 4 (a) for spacecraft 1, showing the semi-major axis over the first orbit. The trend is the consequence of the sail oscillation discussed before. The force generated by the sail, in tangential, normal, and out-ofplane orbital components, is represented in Fig. 5 (b). The tangential component is the one producing the increase in semimajor axis. Note that the second well of the tangential acceleration (when the spacecraft travels toward the sun) is longer than the first one (when the spacecraft travels away from the sun), which is explained by considering the angular position of the sail with respect to the direction tangential to the orbit.

To summarize the results, spacecraft 1 achieves about $19 \mathrm{~km}$ of semimajor axis increase (or altitude gain), while spacecraft 2 achieves $35 \mathrm{~km}$, both with negligible change of eccentricity and inclination. 
Table 1 Spacecraft masses and dimensions.

\begin{tabular}{lcc}
\hline \hline Spacecraft & 1 & 2 \\
\hline \hline Boom length, $l, \mathrm{~m}$ & 1 & 2 \\
\hline Base length, $b, \mathrm{~m}$ & 1.5 & 3 \\
\hline Bus mass, $m_{\text {bus }}, \mathrm{kg}$ & 1 & 2 \\
\hline Bus size, $l_{\text {bus }}, \mathrm{cm}$ & 10 & 20 \\
\hline Total mass, $m, \mathrm{~kg}$ & 1.1 & 2.39 \\
\hline \hline
\end{tabular}
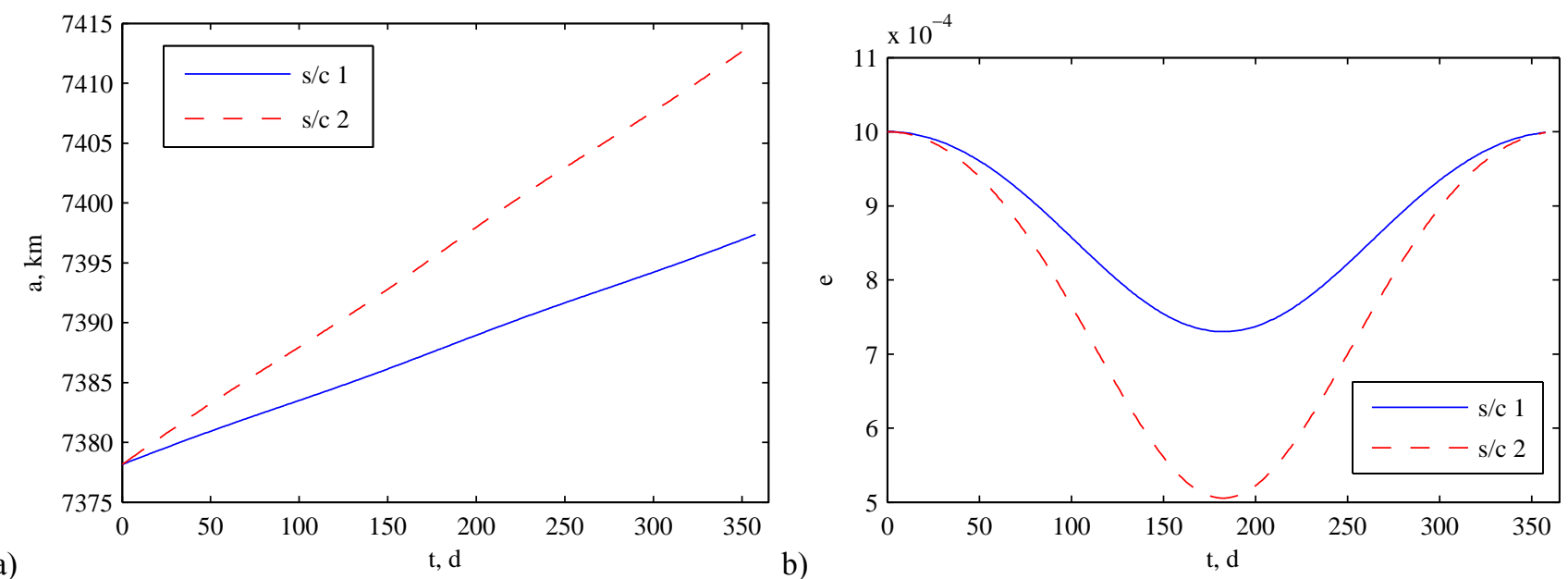

Fig. 4 Semi-major axis (a) and eccentricity (b) over one year for the two spacecraft.
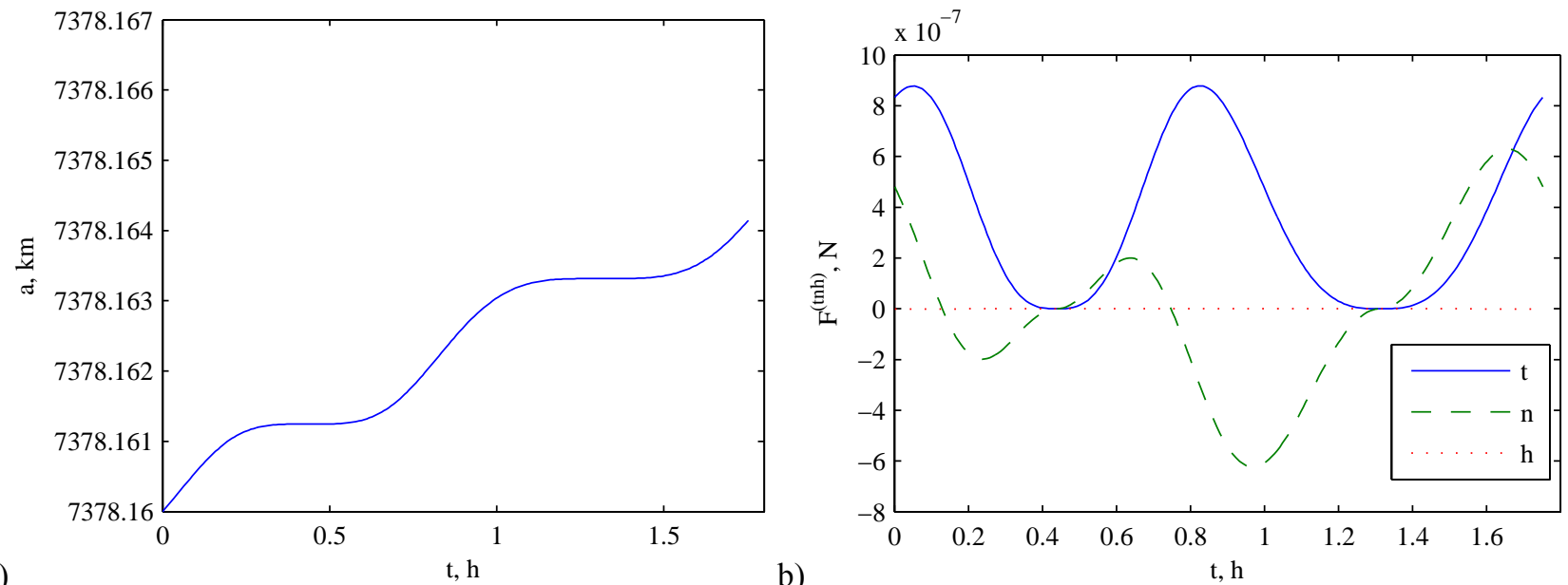

Fig. 5 Spacecraft 1 over the first orbit: (a) Semi-major axis; (b) SRP force in tangential, normal and out-of-plane components.

Fig. 6 shows the control variable, i.e. the moment of inertia around the $x$ axis, at each orbit in the year. There are three visible trends in this plot: the first, is a 1-year-periodic oscillatory motion, which is used to compensate for the declination of the sun over the orbital plane; the second is a slow, quasi-linear increase, which is due to the increase of the orbital semimajor axis; and finally the third is a small oscillation which is due to the controller correcting minor errors in the oscillation at each crossing of the sun-Earth line. The moment of inertia of configuration 1 varies between 0.59 and $0.69 \mathrm{~kg} \mathrm{~m}^{2}$ while 
the second configuration requires a much higher moment of inertia, of order 4.6 to $5.5 \mathrm{~kg} \mathrm{~m}^{2}$. Such high values may be reduced, in practice, by extending a smaller trim-sail on the other side of the bus to oppose the excessive moment of the main sail without opposing the desired force.

A similar, but inverted, trend is found in Fig. 7 (a), which represents the maximum angular velocity (around the $x$ axis) on each orbit for the two spacecraft. Finally, Fig. 7 (b) represents the maximum value of the angle $\theta$, on each orbit, measured with respect to the sun-Earth line (i.e. the amplitude of the oscillation). This starts from the initial value of $\theta_{0}=30^{\circ}$ and it grows up to about $33^{\circ}$. The trend of the curves, which are practically overlapping for the two spacecraft, is due to the adopted control scheme.
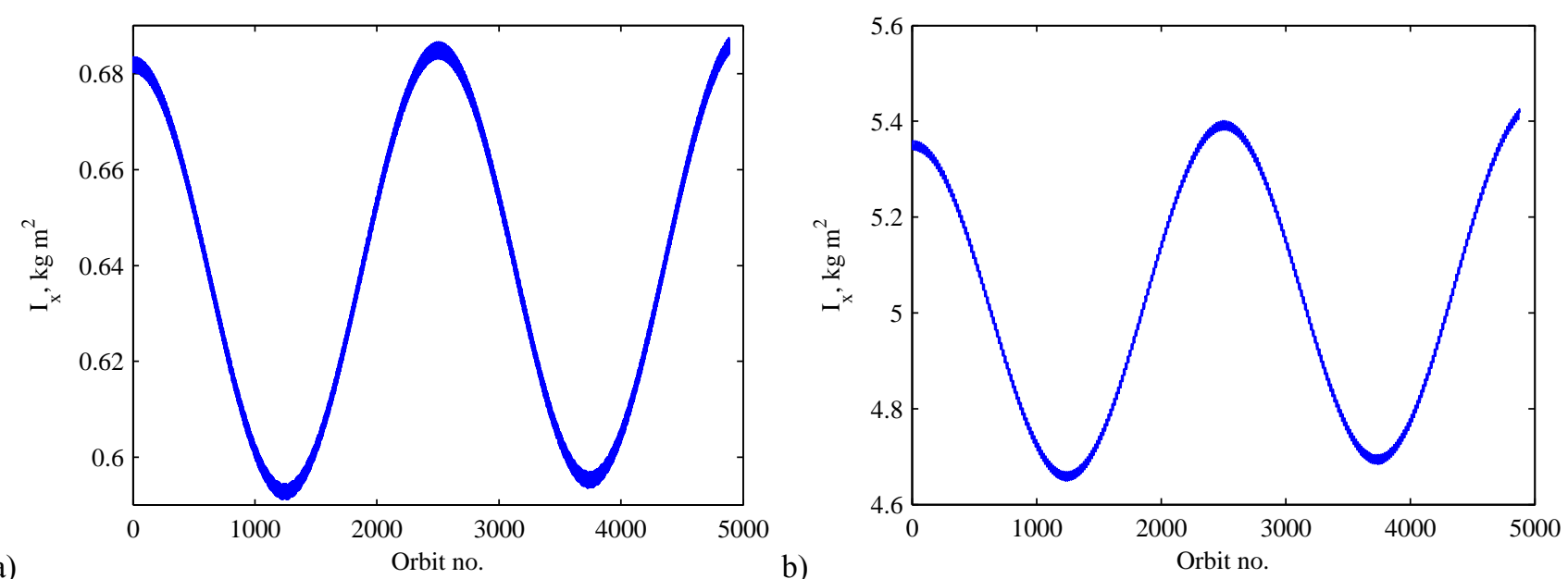

Fig. 6 Moment of inertia over one year for spacecraft 1 (a) and 2 (b).
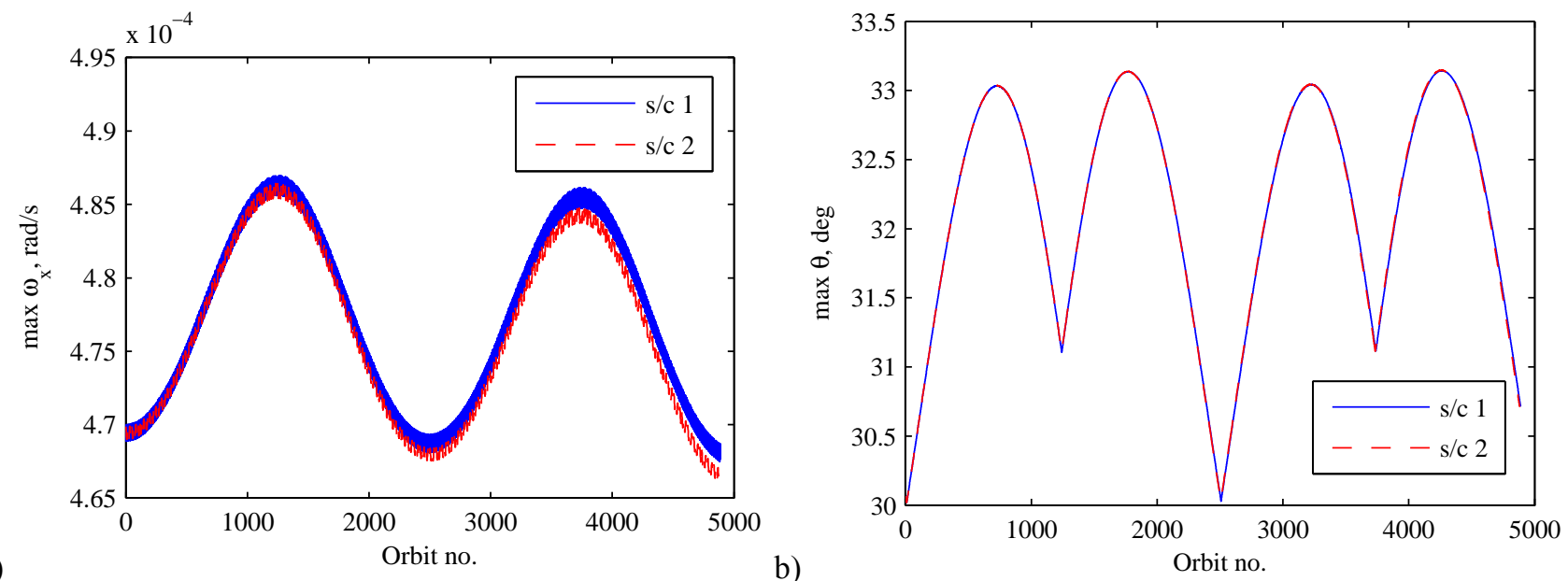

Fig. 7 Maximum angular velocity (a) and amplitude of oscillation (b). 


\section{Conclusion}

A mechanism has been proposed by which a satellite can be induced to oscillate about the sun vector whilst in orbit about the Earth. By adjusting the frequency of these oscillations such that they are synchronized with the orbital period, an SRP-based force can be generated that reverses twice per orbit and thus always acts in the same sense with respect to the velocity vector and either acts to advance or retard the spacecraft accordingly. This effect manifests itself as a change of altitude. Whilst some active control is required to maintain the synchronization of the oscillations and the orbit, this can be achieved by propellantless means such as changes to the rotational inertia of the satellite or changes to the reflectivity of the solar sail, making the change in altitude an essentially free maneuver.

\section{References}

[1] Roberts, P. C. and Harkness, P. G., "Drag Sail for End-of-Life Disposal from Low Earth Orbit," Journal of Spacecraft and Rockets, Vol. 44, No. 6, 2007, pp. 1195-1203.

doi: $10.2514 / 1.28626$

[2] Gloyer, P., Robinson, T., Mignogna, A. and Ahmad, Y., "Aerobraking to Lower Apogee in Earth Orbit with the Small Payload Orbit Transfer (Sport ${ }^{\mathrm{TM}}$ ) Microsatellite Vehicle,” 15th Annual/USU Conference on Small Satellites, Logan, UT, USA, 2001.

[3] Bak, T. W., R., "Passive Aerodynamic Stabilisation of Low Earth Orbit Satellite," Proceedings of the 3rd ESA International Conference on Spacecraft Guidance, Navigation and Control Systems, ESA, Noordwijk, The Netherlands, 1996.

[4] Ceriotti, M., Harkness, P. and McRobb, M., "Variable-Geometry Solar Sailing: The Possibilities of the Quasi-Rhombic Pyramid," $3^{\text {rd }}$ International Symposium on Solar Sailing (ISSS 2013), Glasgow, UK, 2013.

[5] Macdonald, M. and McInnes, C. R., “Analytical Control Laws for Planet-Centred Solar Sailing,” Journal of Guidance, Control, and Dynamics, Vol. 28, No. 5, 2005, pp. 1038-1048.

doi: $10.2514 / 1.11400$

[6] Stolbunov, V., Ceriotti, M., Colombo, C. and McInnes, C. R., "Optimal Law for Inclination Change in an Atmosphere through Solar Sailing," Journal of Guidance, Control, and Dynamics, in press. doi: 10.2514/1.59931

[7] Mengali, G. and Quarta, A. A., "Near-Optimal Solar-Sail Orbit-Raising from Low Earth Orbit," Journal of Spacecraft and Rockets, Vol. 42, No. 5, 2005, pp. 954-958.

doi: $10.2514 / 1.14184$

[8] Macdonald, M. and McInnes, C. R., "Realistic Earth Escape Strategies for Solar Sailing," Journal of Guidance, Control, and Dynamics, Vol. 28, No. 2, 2005, pp. 315-323.

doi: $10.2514 / 1.5165$

[9] Iñarrea, M., Lanchares, V., Palacián, J. F., Pascual, A. I., Salas, J. P. and Yanguas, P., "Chaos and Its Control in the Libration Motion of a Non-Rigid Spacecraft with Viscous Drag in Circular Orbit," Monografias de la Real Academia de Ciencias de Zaragoza, Vol. 28, 2006, pp. 73-84.

[10] Venkatachalam, R., "Large Angle Pitch Attitude Maneuver of a Satellite Using Solar Radiation Pressure," IEEE Transactions on Aerospace and Electronic Systems, Vol. 29, No. 4, 1993, pp. 1164-1169. doi: 10.1109/7.259519

[11] Tsuda, Y., Mori, O., Funase, R., Sawada, H., Yamamoto, T., Saiki, T., Endo, T. and Kawaguchi, J. i., "Flight Status of Ikaros Deep Space Solar Sail Demonstrator," Acta Astronautica, Vol. 69, 2011, pp. 833-840. doi: 10.1016/j.actaastro.2011.06.005

[12] McInnes, C. R., Solar Sailing: Technology, Dynamics and Mission Applications, Springer-Praxis Books in Astronautical Engineering, Springer-Verlag, Berlin, 1999.

[13] Schaub, H. and Junkins, J. L., Analytical Mechanics of Space Systems, 2nd Edition Aiaa Education Series, Reston, VA, USA 2009.

[14] Battin, R. H., An Introduction to the Mathematics and Methods of Astrodynamics, Revised edition, Aiaa Education Series, AIAA, New York, 1999. 